\title{
Water around IRAS 15398-3359 observed with ALMA
}

\author{
P. Bjerkeli ${ }^{1,2}$, J. K. Jørgensen ${ }^{1}$, E. A. Bergin ${ }^{3}$, S. Frimann ${ }^{1}$, D. Harsono ${ }^{4}$, S. K. Jacobsen ${ }^{1}$, J. E. Lindberg ${ }^{5}$, \\ M. Persson ${ }^{4}$, N. Sakai ${ }^{6}$, E. F. van Dishoeck ${ }^{4,7}$, R. Visser ${ }^{3}$, and S. Yamamoto ${ }^{8}$ \\ ${ }^{1}$ Centre for Star and Planet Formation, Niels Bohr Institute \& Natural History Museum of Denmark, University of Copenhagen, \\ Øster Voldgade 5-7, 1350 Copenhagen K., Denmark \\ e-mail: per.bjerkeli@nbi.dk \\ 2 Department of Earth and Space Sciences, Chalmers University of Technology, Onsala Space Observatory, 43992 Onsala, Sweden \\ 3 Department of Astronomy, University of Michigan, 1085 S. University Ave., Ann Arbor, MI 48109-1107, USA \\ ${ }^{4}$ Leiden Observatory, Leiden University, Niels Bohrweg 2, 2333 CA, Leiden, The Netherlands \\ 5 NASA Goddard Space Flight Center, Astrochemistry Laboratory, Mail Code 691, 8800 Greenbelt Road, Greenbelt, MD 20771, \\ USA \\ 6 The Institute of Physical and Chemical Research (RIKEN), 2-1, Hirosawa, Wako-shi, 351-0198 Saitama, Japan \\ 7 Max-Planck-Institut für Extraterrestische Physik, Giessenbachstrasse 2, 85478 Garching, Germany \\ 8 Department of Physics, Graduate School of Science, The University of Tokyo, 7-3-1 Hongo, Bunkyo, 113-0033 Tokyo, Japan
}

Received 26 April 2016 / Accepted 19 July 2016

\begin{abstract}
Context. Understanding how protostars accrete their mass is one of the fundamental problems of star formation. High dust column densities and complex kinematical structures make direct observations challenging. Moreover, direct observations only provide a snapshot. Chemical tracers provide an interesting alternative to characterise the infall histories of protostars.

Aims. We aim to map the distribution and kinematics of gaseous water towards the low-mass embedded protostar IRAS 15398-3359. Previous observations of $\mathrm{H}^{13} \mathrm{CO}^{+}$showed a depression in the abundance towards IRAS 15398-3359. This is a sign of destruction of $\mathrm{HCO}^{+}$by an enhanced presence of gaseous water in an extended region, possibly related to a recent burst in the accretion. Direct observations of water vapour can determine the exact extent of the emission and confirm the hypothesis that $\mathrm{HCO}^{+}$is indeed a good tracer of the water snow-line.

Methods. IRAS 15398-3359 was observed using the Atacama Large Millimeter/submillimeter Array (ALMA) at 0.5" resolution in two setups at 390 and $460 \mathrm{GHz}$. Maps of $\mathrm{HDO}\left(1_{01}-0_{00}\right)$ and $\mathrm{H}_{2}^{18} \mathrm{O}\left(4_{14}-3_{21}\right)$ were taken simultaneously with observations of the CS (87) and $\mathrm{N}_{2} \mathrm{H}^{+}(5-4)$ lines and continuum at 0.65 and $0.75 \mathrm{~mm}$. The maps were interpreted using dust radiative transfer calculations of the protostellar infalling envelope with an outflow cavity.

Results. HDO is clearly detected and extended over the scales of the $\mathrm{H}^{13} \mathrm{CO}^{+}$depression, although it is displaced by $\sim 500 \mathrm{AU}$ in the direction of the outflow. $\mathrm{H}_{2}^{18} \mathrm{O}$ is tentatively detected towards the red-shifted outflow lobe, but otherwise it is absent from the mapped region, which suggests that temperatures are low. Although we cannot entirely exclude a shock origin, this indicates that another process is responsible for the water emission.

Conclusions. Based on the temperature structure obtained from dust radiative transfer models, we conclude that the water was most likely released from the grains in an extended hour-glass configuration during a recent accretion burst. HDO is only detected in the region closest to the protostar, at distances of up to $500 \mathrm{AU}$. These signatures can only be explained if the luminosity has recently been increased by orders of magnitudes. Additionally, the densities in the outflow cones must be sufficiently low.
\end{abstract}

Key words. ISM: individual objects: IRAS 15398 - ISM: molecules - ISM: jets and outflows - stars: winds, outflows stars: formation

\section{Introduction}

When stars form, the bulk of the luminosity is due to the accretion of material onto the protostellar object. It remains a major challenge, however, to directly probe the material as it falls in from the natal cloud. While outflows have been shown to be prominent (Snell et al. 1980), the accretion process is heavily obscured by the dense environments close to protostars. Understanding this process is fundamentally important, however, because it has profound implications on the properties of stars, the initial mass function, and the physical and chemical composition of circumstellar material. In recent years, several studies have focused on the question whether accretion takes place at a constant rate or if violent bursts occur in the accretion rate. Accretion bursts might be the result of fragmentation of protostellar disks (see e.g. Bell \& Lin 1994; Vorobyov \& Basu 2006; Vorobyov et al. 2013) or be related to the nature of the large-scale infall (Padoan et al. 2014). Variations like this are also tied to the so-called luminosity problem (Kenyon et al. 1990), where the observed current luminosities of protostars generally are more than an order of magnitude lower than inferred from standard accretion models (see e.g. Evans et al. 2009). It is therefore possible that protostars spend most of their lifetimes in a quiescent phase and accordingly seem to be less luminous than expected. Bursts in accretion are known to be common and long-lasting in the more evolved FU Orionis stars (Herbig 1977), but it still remains a challenge to determine whether this phenomenon is equally common among younger objects.

Large variations in the accretion rates of protostars may also have important consequences for their chemical structures. To first order, the chemistry is strongly affected by the location where molecules are frozen out on and sublimate from dust 
grains at the so-called snow lines. For a protostar of constant luminosity, the location of these boundaries for the chemistry is well-defined based on how large a region the central protostar can heat up to and above the sublimation temperature of the molecule. However, when a protostar undergoes a burst and significantly increases its luminosity, the dust grains will heat up almost instantly on larger scales (Johnstone et al. 2013), allowing molecules to sublimate from the grain ice-mantles on timescales shorter than a few years (e.g. Rodgers \& Charnley 2003). The opposite process, freeze-out, generally takes a much longer time at the densities characteristic of protostellar envelopes, however: typically $10^{4}-10^{5}$ yr for $\mathrm{CO}$ and $\mathrm{H}_{2} \mathrm{O}$ at the density $n\left(\mathrm{H}_{2}\right)=10^{5} \mathrm{~cm}^{-3}$ and $100-1000 \mathrm{yr}$ at $n\left(\mathrm{H}_{2}\right)=10^{7} \mathrm{~cm}^{-3}$ (e.g. Rodgers \& Charnley 2003). This suggests that observations of even relatively common molecules may provide an indirect way of estimating whether a given protostar has recently undergone a burst in accretion (e.g. Visser \& Bergin 2012; Vorobyov et al. 2013; Visser et al. 2015).

Recent observations (Jørgensen et al. 2015) of a sample of 16 embedded protostars with the SMA show that the extent of the $\mathrm{C}^{18} \mathrm{O}$ emission varies significantly and is not directly correlated with the current luminosities of the protostars. About half the sources show extended $\mathrm{CO}$ emission that would imply that the protostars have undergone bursts within the past $10^{4} \mathrm{yr}$. Freeze-out onto the grains occurs for $\mathrm{CO}$ whenever the temperature drops below $\sim 30 \mathrm{~K}$ (Noble et al. 2012), which typically occurs on relatively large scales in the protostellar envelopes where the density is low and the timescale for freeze-out accordingly is relatively long. To find the sources with the most recent bursts, snow-line tracers at higher densities closer to the protostars are required. Molecules with snow lines at high densities can also trace bursts of higher intensities.

IRAS 15398-3359 (Shirley et al. 2000) is a Class 0 (André et al. 1990, 1993) protostar located in the Lupus I cloud at a distance of $155 \mathrm{pc}$ (Lombardi et al. 2008). The source has a prominent bipolar molecular outflow (Tachihara et al. 1996; van Kempen et al. 2009; Dunham et al. 2014; Y1ld1z et al. 2015; Jørgensen et al. 2013; Oya et al. 2014) that extends less than $3000 \mathrm{AU}$ from the protostar itself, possibly indicating that the source is very young (Bjerkeli et al. 2016). The bolometric luminosity is meassured at $1.8 L_{\odot}$ (Jørgensen et al. 2013), and the source shows a very interesting chemistry. It is one of two known so-called warm carbon-chain chemistry sources (Sakai et al. 2009, 2011). IRAS 15398-3359 was observed with the Atacama Large Millimeter/submillimeter Array (ALMA) in Cycle 0, and these observations revealed a hole in the $\mathrm{H}^{13} \mathrm{CO}^{+}(3-2)$ emission (Jørgensen et al. 2013), while other species (i.e. $\mathrm{C}^{17} \mathrm{O}$ and $\mathrm{CH}_{3} \mathrm{OH}$ ) peak at the central position. As discussed by these authors, the best explanation is an increased abundance of gaseous water due to a recent accretion burst occurring 100-1000 yr ago. In this scenario, the abundance of gaseous water is expected to increase because of the increased stellar luminosity, and the time estimate was based on the expected freeze-out time for water. The abundance of $\mathrm{HCO}^{+}$is expected to decrease whenever water is abundant and temperatures and densities are high through the reactions

$\mathrm{CO}+\mathrm{H}_{3}^{+} \rightarrow \mathrm{HCO}^{+}+\mathrm{H}_{2}$,

$\mathrm{HCO}^{+}+\mathrm{H}_{2} \mathrm{O} \rightarrow \mathrm{H}_{3} \mathrm{O}^{+}+\mathrm{CO}$.

The most surprising aspect of the $\mathrm{H}^{13} \mathrm{CO}^{+}$map presented in Jørgensen et al. (2013) is, however, that the extent of the hole (150-200 AU) is much larger than the region where temperatures are expected to be higher than the sublimation temperature for water, that is, at $100 \mathrm{~K}$. Given the current luminosity of the protostar, the temperature decreases to below $100 \mathrm{~K}$ as close as $\sim 20$ AU (see Sect. 4) from the protostar.

To explain the extent of the $\mathrm{H}^{13} \mathrm{CO}^{+}$absence (and the presence of water), the protostar must have reached a luminosity one to two orders of magnitude higher than its current value. This conclusion is supported by the CO map obtained with the SMA, which reveals dynamical events in the outflow on timescales of a few hundred years (Bjerkeli et al. 2016). An increased accretion rate would probably result in an increased outflow activity during the period of the burst (Raga et al. 1990). However, to confirm this hypothesis, observations of the water molecule itself are required. Since the atmosphere is opaque to the lowestenergy transitions from $\mathrm{H}_{2}^{16} \mathrm{O}$ and because the spatial resolution available with space-based facilities is poor, we here used isotopologues that can be readily observed from the ground. In this program, which was carried out using ALMA during Cycle 2, two isotopologue transitions were targeted to map the distribution of thermal water: $\mathrm{H}_{2}^{18} \mathrm{O}\left(4_{14}-3_{21}\right)$ and $\mathrm{HDO}\left(1_{01}-0_{00}\right)$. These two transitions are complementary in the sense that they probe gas at very different excitation $\left(E_{\text {up }}=390 \mathrm{~K}\right.$ and $\left.E_{\text {up }}=22 \mathrm{~K}\right)$. Whether both HDO and $\mathrm{H}_{2}^{18} \mathrm{O}$ or only HDO is detected therefore depends on the thermal structure of the region.

We discuss the observations of $\mathrm{H}_{2}^{18} \mathrm{O}$ and $\mathrm{HDO}$ in the vicinity of IRAS 15398-3359. We also discuss how the location of the snow line might be affected by the molecular outflow known to be present in the region. The observations and results are presented in Sects. 2 and 3. The emission from water is discussed in Sect. 4, and the results are summarised in Sect. 5.

\section{Observations}

IRAS 15398-3359 was observed using ALMA on 2015 May 20 and 21 as part of the Cycle 2 program 2013.1.00244.S (PI: Jes Jørgensen). The main science goal was to observe the $\operatorname{HDO}\left(1_{01}-0_{00}\right)$ line at $464.925 \mathrm{GHz}$ and the $\mathrm{H}_{2}^{18} \mathrm{O}\left(4_{14}-3_{21}\right)$ line at $390.608 \mathrm{GHz}$. Thirty-six antennas in the $12 \mathrm{~m}$ array were used during the observations, providing baselines in the range $20 \mathrm{~m}(\approx 25-30 \mathrm{k} \lambda)$ to $520 \mathrm{~m}(\approx 700-800 \mathrm{k} \lambda)$. The project was carried out under excellent observing conditions with a precipitable water vapour ranging between $0.26 \mathrm{~mm}$ and $0.41 \mathrm{~mm}$. The phase centre was at $\alpha_{2000}=15^{\mathrm{h}} 43^{\mathrm{m}} 02 \mathrm{~s} 24$, $\delta_{2000}=-34^{\circ} 09^{\prime} 06.7^{\prime \prime}$ and the total observing time was $3.6 \mathrm{~h}$. Two different settings were used to cover both the HDO and the $\mathrm{H}_{2}^{18} \mathrm{O}$ line. The HDO setup contains four line spectral windows centred on $462.607 \mathrm{GHz}, 463.719 \mathrm{GHz}, 464.928 \mathrm{GHz}$, and $465.818 \mathrm{GHz}$, as well as two continuum spectral windows centred on $463.104 \mathrm{GHz}$ and $465.000 \mathrm{GHz}$. The bandwidth for the line spectral windows is $234 \mathrm{MHz}$, and for the continuum spectral windows it is $2000 \mathrm{MHz}$. The corresponding channel spacings are $0.122 \mathrm{MHz}$ and $15.625 \mathrm{MHz}$, respectively. The $\mathrm{H}_{2}^{18} \mathrm{O}$ observation contains three line spectral windows centred on $388.616 \mathrm{GHz}, 390.612 \mathrm{GHz}$, and $391.851 \mathrm{GHz}$, and two continuum spectral windows centred on $401.104 \mathrm{GHz}$ and $402.904 \mathrm{GHz}$. The bandwidths and channel spacings are the same as for the HDO setup. In the settings described above, $\mathrm{N}_{2} \mathrm{H}^{+}(5-4), \mathrm{CS}(8-7)$, and $\mathrm{C}^{33} \mathrm{~S}(8-7)$ were covered as well. The observations are summarised in Table 1.

The data reduction was carried out in CASA (McMullin et al. 2007) and followed the standard procedure. The subsequent analysis of the maps was made in MATLAB. The phase calibration was carried out through observations of the BL Lac object J1517-2422, bandpass calibration on the quasar J1256-0547, and flux calibration on Titan. One 

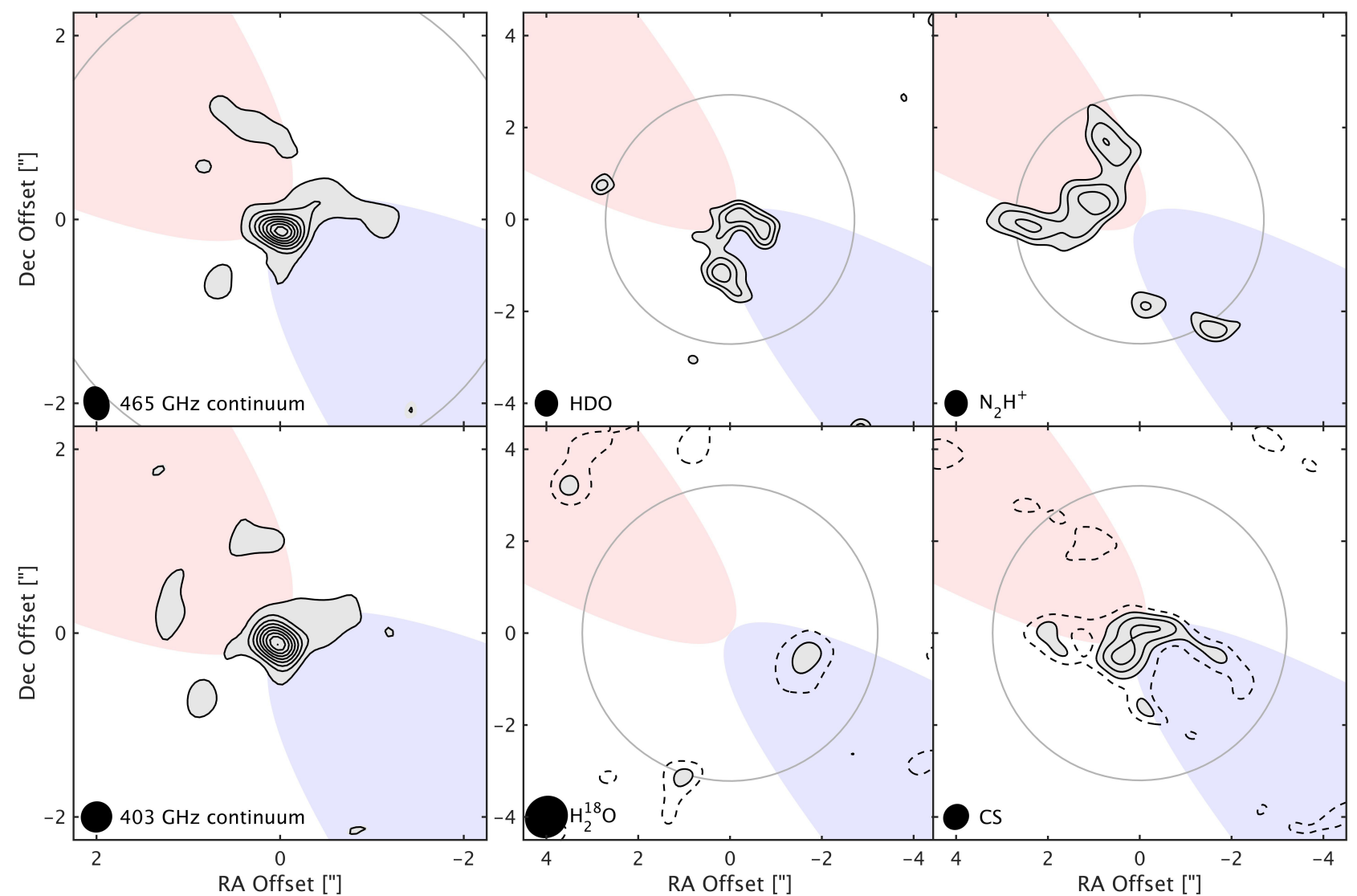

Fig. 1. Continuum and integrated line emission maps towards IRAS $15398-3359$, centred on $\alpha_{2000}=15^{\mathrm{h}} 43^{\mathrm{m}} 02^{\mathrm{s}} \cdot 24, \delta_{2000}=-34^{\circ} 09^{\prime} 06.7^{\prime \prime}$. The lines are integrated over a velocity range of $\Delta v=0.75 \mathrm{~km} \mathrm{~s}^{-1}$ (except for $\mathrm{H}_{2}^{18} \mathrm{O}$, where $\Delta v=2.00 \mathrm{~km} \mathrm{~s}^{-1}$ has been used). Contours are from $3 \sigma$ in steps of $1 \sigma$. For $\mathrm{H}_{2}^{18} \mathrm{O}$ and CS, the $2 \sigma$ level is also indicated with dashed contours. The red and blue shaded regions mark the directions of the outflow cones (same geometry as in the models discussed in Sect. 4.2). The 90-percent level of the beam profile is indicated with a circle in each panel. The beam is shown in the lower left corner of each map. The continuum maps are zoomed-in by a factor of two.

Table 1. ALMA observations.

\begin{tabular}{llllll}
\hline \hline Spectral window & Number of channels/resolution & Center frequency & Bandwidth & Line & $E_{\text {up }}(\mathrm{K})$ \\
\hline HDO setting & & & & & \\
0 & $960 / 244.141 \mathrm{kHz}$ & $465.818 \mathrm{GHz}$ & $234.375 \mathrm{MHz}$ & $\mathrm{N}_{2} \mathrm{H}^{+}(5-4)$ & 67.1 \\
1 & $960 / 244.141 \mathrm{kHz}$ & $464.928 \mathrm{GHz}$ & $234.375 \mathrm{MHz}$ & $\mathrm{HDO}\left(1_{01}-0_{00}\right)$ & 22.3 \\
2 & $128 / 15625.000 \mathrm{kHz}$ & $465.000 \mathrm{GHz}$ & $2000.000 \mathrm{MHz}$ & $\mathrm{Continuum}$ & - \\
$\mathrm{H}_{2}^{18}$ O setting & & & & \\
0 & $960 / 244.141 \mathrm{kHz}$ & $390.612 \mathrm{GHz}$ & $234.375 \mathrm{MHz}$ & $\mathrm{H}_{2}^{18} \mathrm{O}\left(4_{14}-3_{21}\right)$ & 322.0 \\
1 & $960 / 244.141 \mathrm{kHz}$ & $391.851 \mathrm{GHz}$ & $234.375 \mathrm{MHz}$ & $\mathrm{CS}(8-7)$ & 84.6 \\
4 & $128 / 15625.000 \mathrm{kHz}$ & $402.904 \mathrm{GHz}$ & $2000.000 \mathrm{MHz}$ & Continuum & - \\
\hline
\end{tabular}

antenna (DA42) was flagged because of an uncertainty in the position. This has no significant effect on the reduced image cubes and does not alter any of the conclusions presented in this paper, nor does it have a significant effect on the presented maps. The output continuum image cubes have an angular resolution $\sim 0.3^{\prime \prime}$, while the line image cubes have a resolution of $\sim 0.5^{\prime \prime}$. The difference arises because a uv taper was used when CLEANing the lines.

\section{Results}

The key aspect of this program was to image the emission of the two water isopologues, $\mathrm{HDO}$ and $\mathrm{H}_{2}^{18} \mathrm{O}$. The emission maps for these two, along with the other detected species, are presented in Fig. 1.

\section{1. $\mathrm{HDO}$ and $\mathrm{H}_{2}^{18} \mathrm{O}$}

The HDO $\left(1_{01}-0_{00}\right)$ line is detected at the $5 \sigma$ level (integrated flux) in the vicinity of the protostar and in the blue-shifted part of the outflow (see Fig. 1). The spectral location of the line peak intensity is somewhat uncertain because the signal-to-noise ratio is low, but we estimate it to be in the range $v_{\mathrm{LSR}}=5.0-5.5 \mathrm{~km} \mathrm{~s}^{-1}$. The line width is narrow with a $F W H M \simeq 1 \mathrm{~km} \mathrm{~s}^{-1}$. In Fig. 2 the spectra within $2^{\prime \prime}$ of the continuum peak are shown. The HDO 


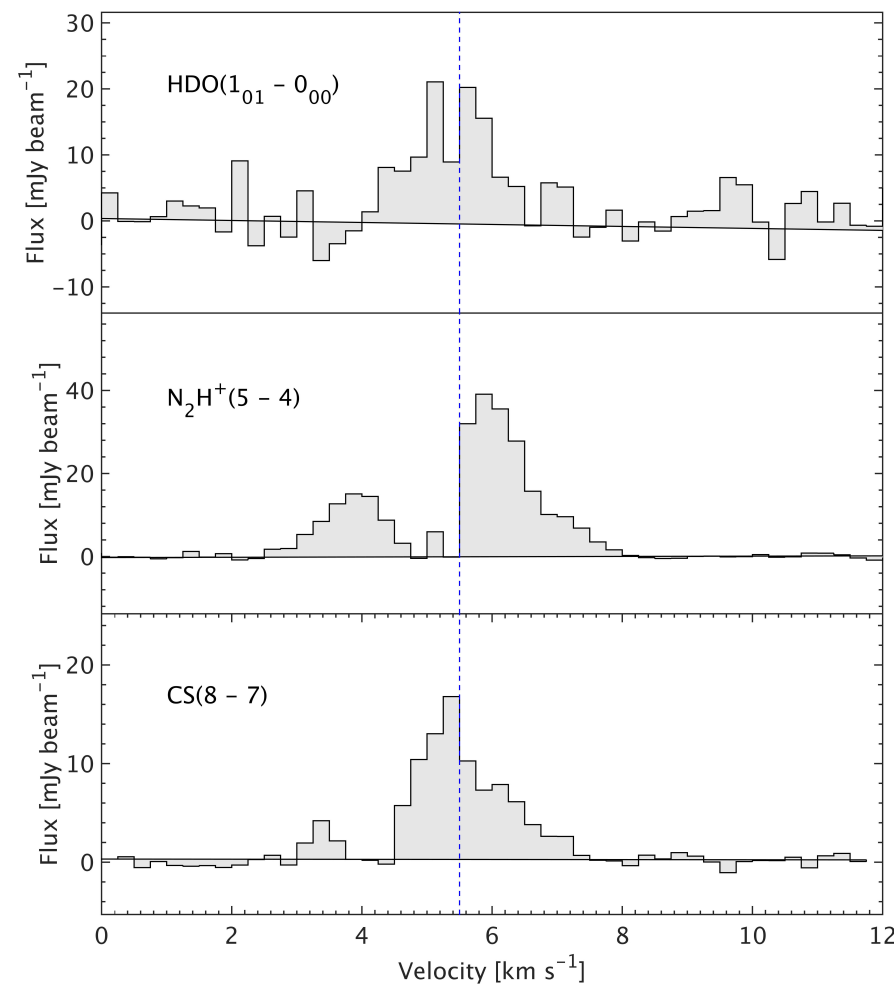

Fig. 2. All spectra within $2^{\prime \prime}\left(1^{\prime \prime}\right.$ for $\left.\operatorname{HDO}\left(1_{01}-0_{00}\right)\right)$ from the centre of the map, averaged together. The vertical dashed line represents the source velocity at $v_{\mathrm{LSR}}=5.5 \mathrm{~km} \mathrm{~s}^{-1}$.

spectrum represents the average of the emission within $1^{\prime \prime}$ from the central position.

$\mathrm{HDO}$ is detected in the region where $\mathrm{H}^{13} \mathrm{CO}^{+}$is not detected (see Fig. 3, and Jørgensen et al. 2013, their Fig. 1), but an extension of the HDO emission towards the south-west of the $\mathrm{H}^{13} \mathrm{CO}^{+}$ ring is visible as well. The extent of this emission is $\sim 300 \mathrm{AU}$ and follow the cavity walls of the blue-shifted outflow outlined by the $\mathrm{CH}_{3} \mathrm{OH}$ emission. Furthermore, a red-shifted component is observed in the red-shifted outflow lobe at the $4 \sigma$ level. This feature is spatially coincident with the emission knot observed in high-velocity CO (2-1) (Bjerkeli et al. 2016, their Fig. 3) and the separation to the central source is $\sim 500 \mathrm{AU}$. In Fig. 4 we plot the $3.5 \sigma$ level contours only for emission integrated over different velocity intervals. Emission at higher velocity with respect to the systemic velocity are indicated in this figure with darker red and blue than the lower velocity emission. Comparing different velocity bins in this way shows a possible gradient in the mapped region. In general, emission at higher velocities is detected at greater distances from the source than at lower velocities.

The $\mathrm{H}_{2}^{18} \mathrm{O}\left(4_{14}-3_{21}\right)$ line in contrast to HDO is not detected towards the protostar. There are two tentative features in the outflow lobes, but only at the $3 \sigma$ level. The north-eastern of these emission knots is located downstream of the feature detected in $\operatorname{HDO}\left(1_{01}-0_{00}\right)$.

\subsection{Continuum and other lines}

The continuum is detected with a peak flux of $36 \pm 1 \mathrm{mJy}^{\text {beam }}{ }^{-1}$ at $465 \mathrm{GHz}$ and $23 \pm 1 \mathrm{mJy}$ beam $^{-1}$ at $403 \mathrm{GHz}$, towards $\alpha_{2000}=15^{\mathrm{h}} 43^{\mathrm{m}} 02 \mathrm{~s} 24, \delta_{2000}=-34^{\circ} 09^{\prime} 06.8^{\prime \prime}$ (from a Gaussian fit). This can be compared to the peak flux of $19 \mathrm{mJy}^{\mathrm{beam}}{ }^{-1}$, reported by Jørgensen et al. (2013) at $345 \mathrm{GHz}$, where the beam size was similar. The largest recoverable scales in the

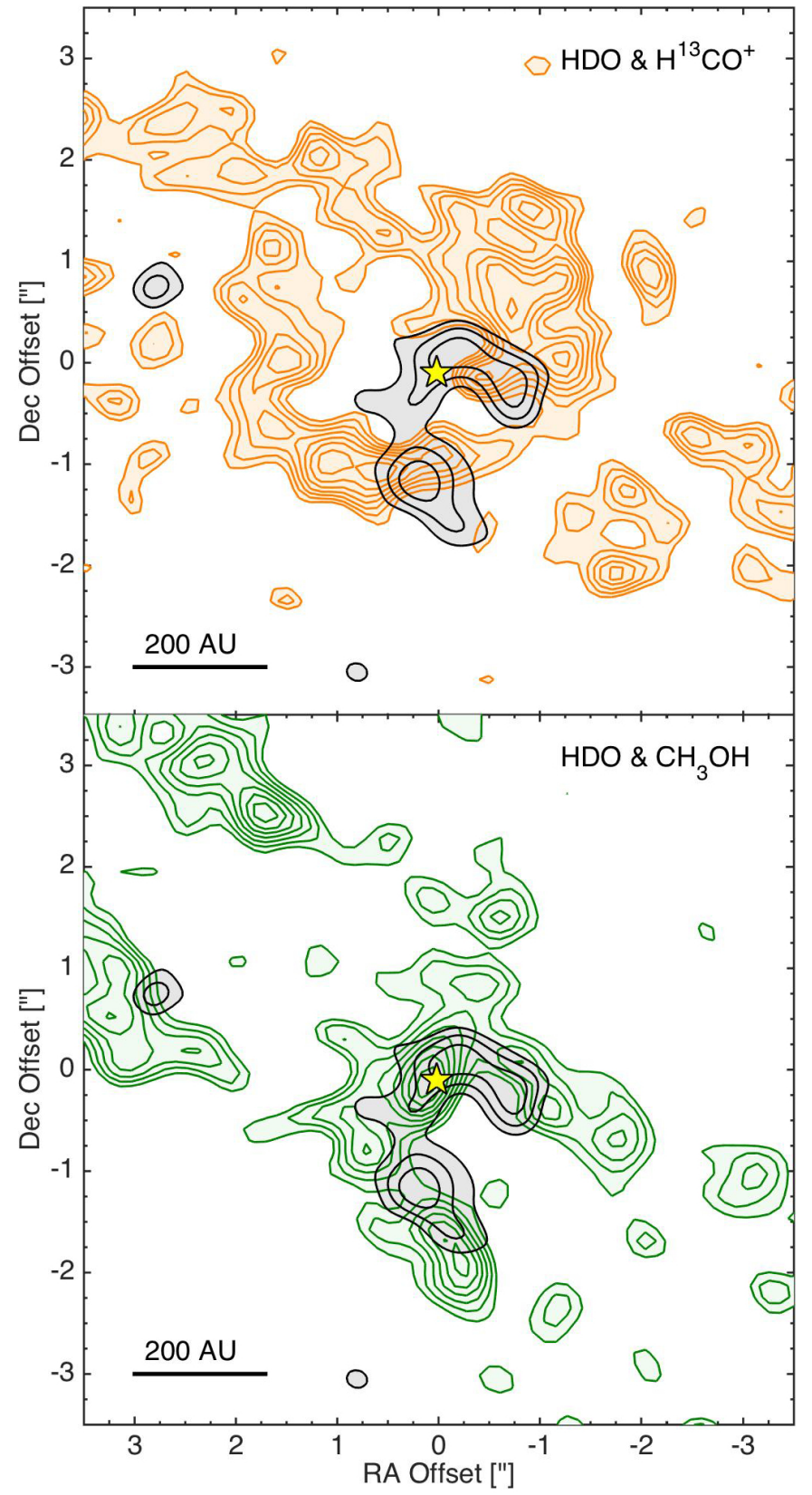

Fig. 3. $\mathrm{HDO}$ emission (black) compared to the $\mathrm{H}^{13} \mathrm{CO}^{+}$emission (brown, upper panel) and the $\mathrm{CH}_{3} \mathrm{OH}$ emission (green, lower panel) presented in Jørgensen et al. (2013). Contours are from $3 \sigma$ in steps on $1 \sigma$. The continuum peak is indicated with a star.

observations presented here range from $4-5^{\prime \prime}$. We therefore cannot exclude that emission from the ambient envelope is resolved out, and some low-level extended emission is observed. The lowest contours of the emission presented in Fig. 1 should therefore be considered as unreliable because we lack short spacing data.

The $\mathrm{N}_{2} \mathrm{H}^{+}(5-4)$ line at $465.825 \mathrm{GHz}$ is detected towards the cavity walls in the red-shifted outflow lobe and in a series of knots in the cavity wall and inner regions of the blue-shifted outflow lobe. No emission is detected towards the protostar itself. A clear velocity gradient is visible in this dataset, and higher velocities are detected farther away from the central source than close to the outflow launching region (Fig. 4).

CS (8-7) is detected towards IRAS 15398-3359, and the emission shows a flattened, slightly elongated structure. CS, at slightly shifted velocities (with respect to $v_{\text {LSR }}=5.5 \mathrm{~km} \mathrm{~s}^{-1}$ ), 

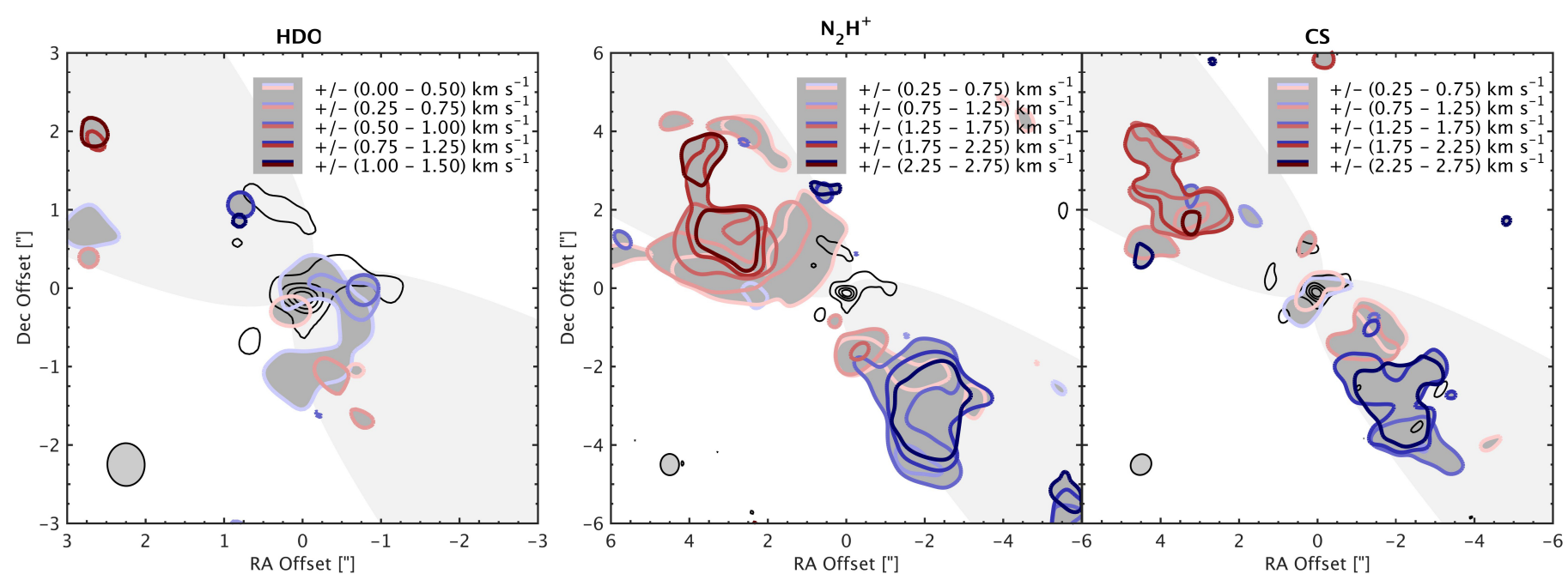

Fig. 4. 3.5 $\sigma$ level in different velocity intervals offset from the systemic velocity (see inset). For HDO, the lowest velocities are towards the central region and slightly displaced into the blue-shifted outflow. Higher velocities are detected further out in both outflow lobes. The $465 \mathrm{GHz}$ (for $\mathrm{HDO}$ ) and $403 \mathrm{GHz}$ (for CS and $\mathrm{N}_{2} \mathrm{H}^{+}$) continuum is plotted with black contours. The extent of the outflow is indicated in gray.

presents two emission regions. As for HDO, the red-shifted region is spatially coincident with the inner emission peak for high-velocity CO (2-1) (Bjerkeli et al. 2016). The same situation applies to the blue-shifted emission, which is spatially coincident with the high-velocity $\mathrm{CO}(2-1)$ blue-shifted knot. This map is presented in Fig. 4, and the emission reveals a velocity structure towards the protostar that weakly indicates rotation. The size of this structure is about $200 \mathrm{AU}$.

The spectra of $\mathrm{N}_{2} \mathrm{H}^{+}$and $\mathrm{CS}$ averaged within $2^{\prime \prime}$ from the central position are presented in Fig. 2. A detailed study of the distribution of the $\mathrm{N}_{2} \mathrm{H}^{+}(5-4)$ and $\mathrm{CS}(8-7)$ emission is deferred to a future paper.

The $\mathrm{C}^{33} \mathrm{~S}(8-7)$ transition at $391.851 \mathrm{GHz}$ was also covered in the spectral setup, but was not detected at an rms level of $\sim 15 \mathrm{mJy}^{\text {beam }^{-1}}$.

\section{Analysis and discussion}

As noted in the previous section, HDO is clearly detected in the inner region and is also displaced into the outflow lobes compared to the centre of the $\mathrm{H}^{13} \mathrm{CO}^{+}$ring, while $\mathrm{H}_{2}^{18} \mathrm{O}$ is not detected. A comparison between $\mathrm{H}^{13} \mathrm{CO}^{+}$and HDO is shown in Fig. 3. Parts of the HDO emission originate in the region where $\mathrm{H}^{13} \mathrm{CO}^{+}$is not detected, although the extent does not cover the full size of the $\mathrm{H}^{13} \mathrm{CO}^{+}$hole. On the other hand, HDO is also abundant in the outflow region where $\mathrm{H}^{13} \mathrm{CO}^{+}$is detected. The low-level HDO emission is not present in the red-shifted outflow close to the central protostar because the short-wavelength dust emission is optically thick and naturally blocks the red-shifted emission at the far side of the protostar. HDO is detected in the blue-shifted outflow lobe while $\mathrm{H}^{13} \mathrm{CO}^{+}$is not; this may be a geometrical effect. $\mathrm{HCO}^{+}$is known to trace the outflow cavity walls (Bjerkeli et al. 2016), and we cannot exclude from the maps alone that HDO might be tracing a region inside of the $\mathrm{HCO}^{+}$emission. The fact that HDO is present towards the continuum peak supports the scenario outlined by Jørgensen et al. (2013). However, the apparent association with the outflow lobes observed here needs an explanation as well. There may be several causes of this spatial shift and the origin of the HDO emission, as we discuss in the sections below.

\subsection{Water chemistry in shocked outflowing gas?}

Since the HDO emission is spatially coincident with the outflowing gas, it is reasonable to assume that high-temperature chemistry plays an important role in this case. At high temperatures and densities, neutral-neutral reactions in the gas phase are efficient in forming water, but frozen water can also easily be desorbed from grains (see e.g. van Dishoeck et al. 2014, for a recent review on the topic). These are conditions that are known to be present in molecular outflows and shocks (see e.g. Tafalla et al. 2013).

Using RADEX and assuming relative abundances with respect to $\mathrm{H}_{2}$ of $X(\mathrm{HDO})=2 \times 10^{-7}$ (Persson et al. 2014) and $X\left(\mathrm{H}_{2}^{18} \mathrm{O}\right)=1 \times 10^{-7}$ (Wilson \& Rood 1994), we estimate that the gas thermal pressure, $n T$, needs to be as high as $10^{11} \mathrm{~cm}^{-3} \mathrm{~K}$ to excite the $\mathrm{H}_{2}^{18} \mathrm{O}\left(4_{14}-3_{21}\right)$ line at the 3 -sigma level, given the strength of the HDO $\left(1_{01}-0_{00}\right)$ line. The reason for this is the different upper state energy of the lines (i.e. HDO at $22 \mathrm{~K}$ and $\mathrm{H}_{2}^{18} \mathrm{O}$ at $390 \mathrm{~K}$ ), which essentially constrains the temperature of the gas. High densities and temperatures can be found in shocks and in hot corinos, but they are less likely inside the outflow cones. The non-detection of $\mathrm{H}_{2}^{18} \mathrm{O}$ therefore tells us that the pressure cannot be too high, not even close to the protostar itself. However, it does not exclude a shock origin. Previous studies suggested that $\mathrm{H}_{2}$ densities are indeed high in shocked regions, although generally not above $10^{8} \mathrm{~cm}^{-3}$ (Mottram et al. 2014).

An even stronger argument against the shock scenario is instead the narrow width of the HDO line $\left(\Delta v \simeq 1 \mathrm{~km} \mathrm{~s}^{-1}\right)$. In shocks, much higher velocities are expected for water (see e.g. Kristensen et al. 2012). Even if the low-velocity HDO is due to sputtering from the ice (Suutarinen et al. 2014) and not to gasphase reactions in the post-shock gas where the $\mathrm{HD} / \mathrm{H}_{2}$ abundance can be significantly decreased (Neufeld et al. 2006), we would not expect the lines to be as narrow as $\sim 1 \mathrm{~km} \mathrm{~s}^{-1}$. Moreover, a shock origin does not explain why HDO is only detected in the region closest to the protostar. If shock chemistry were the sole explanation to the emission, then there is no reason why we should not also observe HDO at larger distances.

Downstream of the red-shifted knot (Fig. 1), a tentative $\mathrm{H}_{2}^{18} \mathrm{O}$ feature is visible. Although we can at present not confirm that it is real, it prohibits us from drawing firm conclusions about 


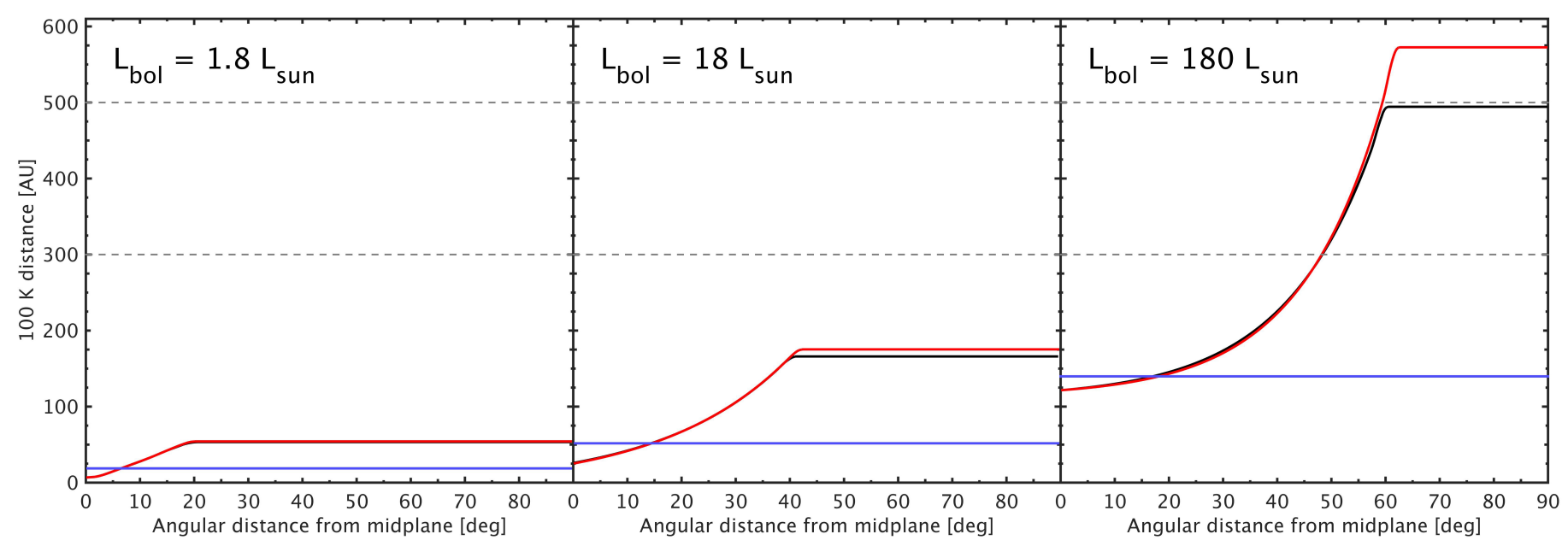

Fig. 5. $100 \mathrm{~K}$ distance from the protostar as a function of the angle to the mid-plane of the system. The curves show the distance at which the temperature drops below $100 \mathrm{~K}$ for three different scenarios. In the first scenario (blue), a pure envelope density profile is considered. In the second scenario (black), an outflow cone with a constant density at $10^{5} \mathrm{~cm}^{-3}$ is added to the model. The third scenario (red) is identical to the second, with the exception that the outflow density is lower, $10^{4} \mathrm{~cm}^{-3}$. The left panel shows the results for a protostar with $L_{\mathrm{bol}}=1.8 L_{\odot}$. The middle and right panels show the same curves when the luminosity is increased by one and two orders of magnitude, respectively. Dashed grey lines indicate the largest distance from the continuum peak for blue-shifted HDO emission ( $\sim 300 \mathrm{AU})$ and red-shifted emission $(\sim 500 \mathrm{AU})$.

the shock chemistry in this particular region. From these data we cannot exclude a very high pressure.

\subsection{Ice sublimation during a recent accretion burst?}

Another possible explanation of the distribution and kinematics of the HDO emission is that water was released from the grains during a recent accretion burst (i.e. the scenario outlined in Jørgensen et al. 2013). If the water release from the grains were due to an increase in luminosity, then how close to the protostar did this occur? As mentioned before, the extent of the HDO emission is several hundred AUs.

\subsubsection{Water sublimation in spherical envelope}

To test this scenario for a purely spherical envelope, we constructed a model of the IRAS 15398-3359 system using RADMC-3D ${ }^{1}$ (Dullemond \& Dominik 2004). This code uses the method described by Bjorkman \& Wood (2001) to calculate the dust temperature distribution in a pre-defined geometry. In this work, fifty million photon packets were propagated through the model where the infalling envelope density profile was taken from the best-fit model presented in Mottram et al. (2013) and followed a power law with an exponent $p=-1.4$, where $n_{\mathrm{H}_{2}}=2 \times 10^{9} \mathrm{~cm}^{-3}$ at $6.1 \mathrm{AU}$. The spherical model has a radius of $1000 \mathrm{AU}$ so that it covers the region from which the emission was analysed. One thousand grid points in the outflow direction were used, and the spacing between them was logarithmic, meaning that fewer grid points were used at larger radii. One thousand grid points were also used in the azimuthal direction, evenly distributed in this case. The dust-togas mass ratio was taken to be 100 . A recent study towards the $\rho$ Oph A region showed that this ratio can vary by at least a factor of 2 (Liseau et al. 2015). This does not affect the results presented here, however, since the densities we used are assumptions and not actual estimates. No scattering was used here, and

\footnotetext{
1 http://www.ita.uni-heidelberg.de/ dullemond/ software/radmc-3d/
}

tests showed that this assumption does not alter our conclusions. Using anisotropic scattering will not change the distances reported here by more than $10 \%$.

The results from these calculations are presented in Fig. 5. The $100 \mathrm{~K}$ distance is plotted (blue lines) as a function of angular distance from the axis perpendicular to the direction of the outflow. For a spherical envelope, this is a straight line, since the temperature only depends on the radius. Three different cases were considered, one with the same luminosity as the current luminosity of the protostar, and two cases with a luminosity increased by one and two orders of magnitude, respectively. It is clear that an envelope profile cannot explain the large extent of the HDO emission. Not even for the case when the luminosity is increased by two orders of magnitude is the $100 \mathrm{~K}$ radius farther away than $150 \mathrm{AU}$ from the protostar.

\subsubsection{Water sublimation in spherical envelope, displaced by outflow motions}

Another possibility is that the gas has been displaced outwards by the motion of the outflow. In that case, and during the lifetime of the source, the extent of the water-emitting region would increase. Although it is difficult in this scenario to explain that the $\mathrm{H}^{13} \mathrm{CO}^{+}$ring is centred on the continuum peak, we cannot exclude it from the water data alone. One major problem with this scenario, however, is that the detected highest velocities are fairly small in comparison. The highest velocity of the HDO emission is $\sim 1 \mathrm{~km} \mathrm{~s}^{-1}$ with respect to the systemic velocity. Although there can be gas moving at higher velocities, the bulk part of the HDO emitting gas must move slowly. At the current velocity, this gives us a timescale of $\sim 2300 \mathrm{yr}$ for the gas to reach the location of the red-shifted knot and $\sim 1600$ yr to reach the farthest extent of the blue-shifted emission. These timescales are both too long compared to the estimated timescale of the most recent accretion burst (100-1000 yr ago, Jørgensen et al. 2013). Even if we (given the low signal-to-noise ratio attained for HDO) assume that this is an underestimation by a factor of two, velocities are still on the lower end. We therefore conclude that this is most likely not the main cause of the extended emission. 


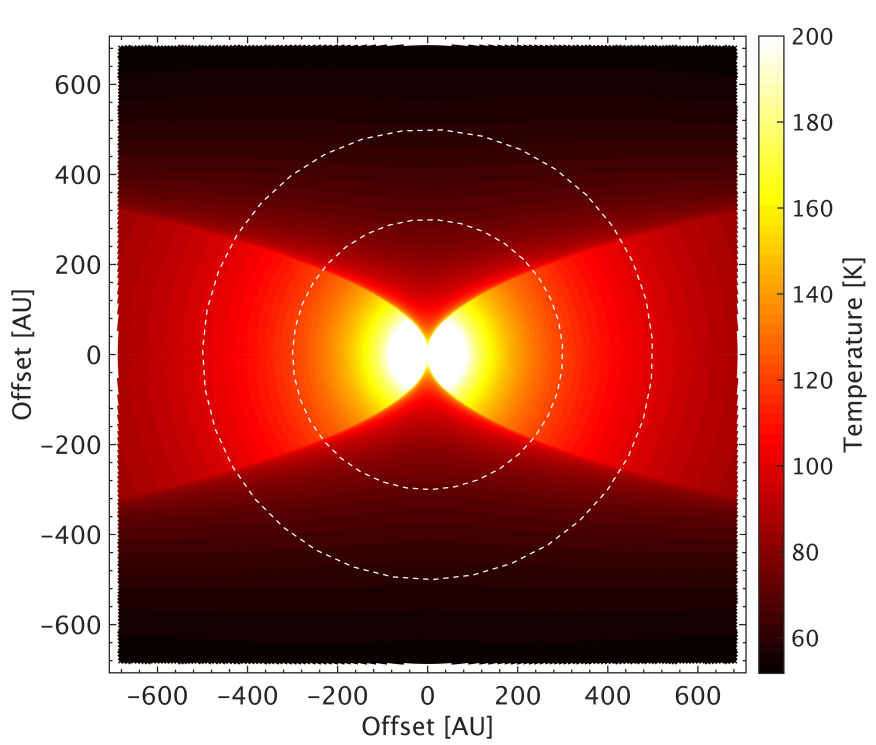

Fig. 6. Temperature map of the region when the density in the outflow cavity is constant at $n_{\mathrm{H}_{2}}=10^{5} \mathrm{~cm}^{-3}$ and the luminosity of the protostar is increased by two orders of magnitude, i.e. $180 L_{\odot}$. Only the region with a temperature lower than $200 \mathrm{~K}$ is presented. Dashed white lines indicate the largest distance from the continuum peak for blue-shifted HDO emission ( 300 AU) and red-shifted emission ( 500 AU).

\subsubsection{Water sublimation in low-density outflow}

Yet another possibility is that water was released from the grains at the time when the protostar underwent a luminosity burst, but that the distance out to where this occurred was changed significantly compared to what is expected for a simple power-law density profile. If densities are lower in the inner parts of the outflow cavity, then the $100 \mathrm{~K}$ radius can be changed significantly compared to the case where only an envelope is present. To test that, we adopted a model similar to the one presented in Bjerkeli et al. (2016, Model M3). The geometry of the model presented in that paper resembles the structure of the emission and the observed line profiles well, both the large-scale $\mathrm{CO}$ outflow and the small-scale $\mathrm{C}_{2} \mathrm{H}$ emission, tracing the outflow cavity walls (Jørgensen et al. 2013). The infalling envelope density profile in this case is the same as before, but we also included an outflow that is described by a wind-driven shell (Lee et al. 2000, their Fig. 21). The details of this model can be found in Bjerkeli et al. (2016). Based on the available data towards IRAS 15398-3359 we cannot estimate the density of the gas inside the outflow cone, and we therefore tested different density distributions (envelope and outflow where $n_{\mathrm{H}_{2}}=10^{5} \mathrm{~cm}^{-3}$, and envelope and outflow where $n_{\mathrm{H}_{2}}=10^{4} \mathrm{~cm}^{-3}$ ) to investigate how this affects the distance to which the temperature drops below $100 \mathrm{~K}$. These scenarios are both consistent with the non-detection of $\mathrm{H}_{2}^{18} \mathrm{O}$. For a temperature at $100 \mathrm{~K}$ and a density of $10^{5} \mathrm{~cm}^{-3}$, the strength of the $\mathrm{H}_{2}^{18} \mathrm{O}\left(4_{14}-3_{21}\right)$ line should be a factor of $\sim 10^{5}$ lower than for the HDO $\left(1_{01}-0_{00}\right)$ line. A temperature map of the region when the outflow density is constant at $n_{\mathrm{H}_{2}}=10^{5} \mathrm{~cm}^{-3}$ and the luminosity of the protostar is increased by two orders of magnitude is presented in Fig. 6.

This figure clearly shows that if the density in the outflow cavity is lower than in the envelope at equal distances from the protostar, then the $100 \mathrm{~K}$ radius is indeed displaced to larger radii. A similar map was also created (but not presented here) for the case where the outflow density is even lower, $n_{\mathrm{H}_{2}}=10^{4} \mathrm{~cm}^{-3}$. The inclination of the outflow with respect to the plane of the sky, which has been estimated to be $20^{\circ}$ (Oya et al. 2014), does not alter these numbers by more than $6 \%$. Owing to the lower density in the outflow, there is in these cases an angular dependence on the $100 \mathrm{~K}$ distance, and this is presented for the two outflow scenarios along with the envelope-only scenario in Fig. 5. It is obvious that for the $100 \mathrm{~K}$ radius to be as far out as $500 \mathrm{AU}$, a high-luminosity central heating is required that would be 10 100 times of its current luminosity. In addition, a relatively low outflow density is required, $n\left(\mathrm{H}_{2}\right)<1 \times 10^{5} \mathrm{~cm}^{-3}$.

\section{Conclusions}

From the observations presented in this paper we conclude the following:

- $\operatorname{HDO}\left(1_{01}-0_{00}\right)$ at $464.925 \mathrm{GHz}$ is detected towards IRAS 15398-3359 and peaks at the $5 \sigma$ level (integrated emission). $\mathrm{H}_{2}^{18} \mathrm{O}\left(4_{14}-3_{21}\right)$, on the other hand, is not detected, which implies a pressure lower than $\sim 10^{11} \mathrm{~cm}^{-3} \mathrm{~K}$. The presence of water in the region where $\mathrm{H}^{13} \mathrm{CO}^{+}$is not detected, is consistent with a recent burst scenario where $\mathrm{HCO}^{+}$is destroyed through reactions with water.

- The morphology of the HDO emission shows that water is present in the outflow as well, but not at distances farther away than $\sim 500$ AU from the protostar. Although we cannot exclude the possibility that shocked regions play a role at a lower level, the observed velocities and spatial distribution are not easily reconcilable with shock chemistry. It is also unlikely that the water was released from the grains close to the protostar and transported outwards by the outflow.

- RADMC-3D models of a geometry containing an infalling envelope and an outflow at lower density showed that the $100 \mathrm{~K}$ distance from the protostar can be displaced outwards to $\sim 500 \mathrm{AU}$. The same models show that the $100 \mathrm{~K}$ distance is within $150 \mathrm{AU}$ from the protostar when the outflow has the same density distribution as the envelope. We therefore find it most likely that the water was released from the grains in an extended hour-glass configuration during a recent accretion burst.

- In addition to the targeted science lines, $\mathrm{N}_{2} \mathrm{H}^{+}(5-4)$ and CS (8-7) were also detected in the mapped region within the same setup. $\mathrm{N}_{2} \mathrm{H}^{+}$traces the outflow cavity walls and shows a rich velocity structure, while CS mainly traces the shocked regions and the central condensation.

To summarise, these data demonstrate the use of imaging molecular emission as a tracer of the evolutionary histories of embedded protostars. Although care must be taken when separating the various components of the protostellar systems (e.g. envelopes, disks, and outflows), the high angular resolution images of ALMA provide the necessary direct constraints. Studies of the molecular emission towards protostars combined with detailed modelling efforts (e.g. chemical and radiative transfer) will be an important tool in the future, in particular when larger samples of sources are systematically targeted in similar lines.

Acknowledgements. This paper makes use of the following ALMA data: ADS/JAO.ALMA\#2013.1.00244.S and \#2011.0.00628.S. ALMA is a partnership of ESO (representing its member states), NSF (USA) and NINS (Japan), together with NRC (Canada), NSC and ASIAA (Taiwan), and KASI (Republic of Korea), in cooperation with the Republic of Chile. The Joint ALMA Observatory is operated by ESO, AUI/NRAO and NAOJ. We also thank Ivan MartiVidal, Wouter Vlemmings and the staff at the Nordic ARC node in Onsala for valuable support in reducing the dataset presented in this paper. This research was supported by the Swedish Research Council (VR) through the contract 637 2013-472 to Per Bjerkeli. Jes Jørgensen acknowledges support by a Lundbeck 
Foundation Junior Group Leader Fellowship as well as the European Research Council (ERC) under the European Union's Horizon 2020 research and innovation programme (grant agreement No. 646908) through ERC Consolidator Grant "S4F". The Centre for Star and Planet Formation is funded by the Danish National Research Foundation. Daniel Harsono is funded by Deutsche Forschungsgemeinschaft Schwerpunktprogramm (DFG SPP 1385) "The First 10 Million Years of the Solar System - a Planetary Materials Approach".

\section{References}

André, P., Martin-Pintado, J., Despois, D., \& Montmerle, T. 1990, A\&A, 236, 180

André, P., Ward-Thompson, D., \& Barsony, M. 1993, ApJ, 406, 122

Bell, K. R., \& Lin, D. N. C. 1994, ApJ, 427, 987

Bjerkeli, P., Jørgensen, J. K., \& Brinch, C. 2016, A\&A, 587, A145

Bjorkman, J. E., \& Wood, K. 2001, ApJ, 554, 615

Dullemond, C. P., \& Dominik, C. 2004, A\&A, 417, 159

Dunham, M. M., Arce, H. G., Mardones, D., et al. 2014, ApJ, 783, 29

Evans, II, N. J., Dunham, M. M., Jørgensen, J. K., et al. 2009, ApJS, 181, 321

Herbig, G. H. 1977, ApJ, 217, 693

Johnstone, D., Hendricks, B., Herczeg, G. J., \& Bruderer, S. 2013, ApJ, 765, 133

Jørgensen, J. K., Visser, R., Sakai, N., et al. 2013, ApJ, 779, L22

Jørgensen, J. K., Visser, R., Williams, J. P., \& Bergin, E. A. 2015, A\&A, 579, A23

Kenyon, S. J., Hartmann, L. W., Strom, K. M., \& Strom, S. E. 1990, AJ, 99, 869

Kristensen, L. E., van Dishoeck, E. F., Bergin, E. A., et al. 2012, A\&A, 542, A8

Lee, C.-F., Mundy, L. G., Reipurth, B., Ostriker, E. C., \& Stone, J. M. 2000, ApJ, 542,925

Liseau, R., Larsson, B., Lunttila, T., et al. 2015, A\&A, 578, A131

Lombardi, M., Lada, C. J., \& Alves, J. 2008, A\&A, 489, 143

McMullin, J. P., Waters, B., Schiebel, D., Young, W., \& Golap, K. 2007, in Astronomical Data Analysis Software and Systems XVI, eds. R. A. Shaw, F. Hill, \& D. J. Bell, ASP Conf. Ser., 376, 127
Mottram, J. C., van Dishoeck, E. F., Schmalzl, M., et al. 2013, A\&A, 558, A126 Mottram, J. C., Kristensen, L. E., van Dishoeck, E. F., et al. 2014, A\&A, 572, A21

Neufeld, D. A., Green, J. D., Hollenbach, D. J., et al. 2006, ApJ, 647, L33

Noble, J. A., Congiu, E., Dulieu, F., \& Fraser, H. J. 2012, MNRAS, 421, 768

Oya, Y., Sakai, N., Sakai, T., et al. 2014, ApJ, 795, 152

Padoan, P., Haugbølle, T., \& Nordlund, A. 2014, ApJ, 797, 32

Persson, M. V., Jørgensen, J. K., van Dishoeck, E. F., \& Harsono, D. 2014, A\&A, 563, A74

Raga, A. C., Binette, L., Canto, J., \& Calvet, N. 1990, ApJ, 364, 601

Rodgers, S. D., \& Charnley, S. B. 2003, ApJ, 585, 355

Sakai, N., Sakai, T., Hirota, T., Burton, M., \& Yamamoto, S. 2009, ApJ, 697, 769

Sakai, N., Sakai, T., Hirota, T., \& Yamamoto, S. 2011, in EAS Pub. Ser. 52, eds. M. Röllig, R. Simon, V. Ossenkopf, \& J. Stutzki, 235

Shirley, Y. L., Evans, II, N. J., Rawlings, J. M. C., \& Gregersen, E. M. 2000, ApJS, 131, 249

Snell, R. L., Loren, R. B., \& Plambeck, R. L. 1980, ApJ, 239, L17

Suutarinen, A. N., Kristensen, L. E., Mottram, J. C., Fraser, H. J., \& van Dishoeck, E. F. 2014, MNRAS, 440, 1844

Tachihara, K., Dobashi, K., Mizuno, A., Ogawa, H., \& Fukui, Y. 1996, PASJ, 48, 489

Tafalla, M., Liseau, R., Nisini, B., et al. 2013, A\&A, 551, A116

van Dishoeck, E. F., Bergin, E. A., Lis, D. C., \& Lunine, J. I. 2014, Protostars and Planets VI, 835

van Kempen, T. A., van Dishoeck, E. F., Hogerheijde, M. R., \& Güsten, R. 2009, A\&A, 508, 259

Visser, R., \& Bergin, E. A. 2012, ApJ, 754, L18

Visser, R., Bergin, E. A., \& Jørgensen, J. K. 2015, A\&A, 577, A102

Vorobyov, E. I., \& Basu, S. 2006, ApJ, 650, 956

Vorobyov, E. I., DeSouza, A. L., \& Basu, S. 2013, ApJ, 768, 131

Wilson, T. L., \& Rood, R. 1994, ARA\&A, 32, 191

Yildı, U. A., Kristensen, L. E., van Dishoeck, E. F., et al. 2015, A\&A, 576, A109 